\title{
IMPACTO DA SATISFAÇÃO COM O APOIO SOCIAL EM ATLETAS
}

\author{
Ana Moisão \\ University of Aveiro, Portugal \\ ana.karina.teodosio@gmail.com \\ Sónia Brito-Costa \\ Institute of Applied Research of Polytechnic Institute of Coimbra \\ sonya.b.costa@gmail.com \\ Florencio Vicente Castro \\ fvicentec@gmail.com \\ Juan José Maldonado Briegas \\ University of Extremadura \\ juanjose.maldonadob@gmail.com
}

Fecha de Recepción: 13 Junio 2018

Fecha de Admisión: 1 Octubre 2018

\section{RESUMO}

0 objetivo deste estudo foi perceber as relações entre os aspetos psicossociais que favorecem e ou inibem a performance desportiva, sendo a satisfação com o suporte social uma variável de sucesso para o rendimento que pode afetar o desempenho e influenciar os resultados. Utilizou-se a versão Portuguesa da (ESSS) Escala de Satisfação com o Suporte Social (Ribeiro, 1999) numa amostra constituída por 302 atletas séniores de futebol, género masculino, com idades compreendidas entre os 18 e os 38 anos de idade. A análise estatística foi efectuada através do Statistical Package for the Social Sciences (SPSS) versão 20.0 para Windows. 0 estudo revelou que a perceção que 0 atleta tem do suporte social é passível de afetar e influenciar o seu equilíbrio global e a satisfação com o suporte social é influenciada pela idade, lesões ou doenças, escalão competitivo, período de treino, número de treinos semanais, posição de jogo e tempo gasto nas deslocações para 0 treino. Os resultados permitem delinear programas preventivos e de intervenção durante as fases de pré-competição e competição.

Palavras Chave: satisfação; suporte social; desempenho; rendimento

\section{ABSTRACT}

IMPACT OF SATISFACTION WITH SOCIAL SUPPORT IN ATHLETES

The aim of this study was to perceive the relationship between psychosocial aspects that favor and / or inhibit the sporting performance, and satisfaction with social support a successful variable to the income that can affect performance and influence the results. We used the Portuguese ver- 
sion of (ESSS) Satisfaction Scale Social Support (Ribeiro, 1999) and he sample consisted of 302 professional soccer players, males, aged between 18 and 38 years old. Statistical analysis was performed using the Statistical Package for Social Sciences (SPSS) version 20.0 for Windows. The study revealed that the perception that the athlete has about the social support is likely to affect and influence their overall balance and satisfaction with social support is influenced by age, injury or illness, competitive level, training period, number of weekly workouts, playing position and time spent traveling to training. The results alloew to delineate prevention programs and intervention during the pre-competition and competition phases.

Keywords: satisfaction; social support; performance

\section{INTRODUÇÃO}

0 suporte social envolve as relações interpessoais, é um conceito abrangente, principalmente estudado na psicologia da saúde, pelo impacto nefasto que pode resultar a sua carência na vida dos indivíduos. As relações interpessoais determinam a forma como as pessoas lidam e se ajustam às situações de vida diária, com as quais se confrontam (Berkman, 1984; Bruhn \& Philips, 1984; Cassel, 1976; Cobb, 1976; Cohen, 1988; Dunbar, Ford, \& Hunt, 1998; Kaplan, Cassel, \& Goe, 1977; Taylor, 1990).

Parece existir um consenso para a sua definição, considerando todos os recursos disponíveis, respostas obtidas e assistência prestada aos pedidos de ajuda, bem como os aspetos funcionais das relações sociais, para que o indivíduo acredite que é amado, estimado, compreendido, valorizado, e que pertence a uma rede de relações sociais mútuas. É assim definido como todo o suporte fornecido pela rede social em momentos de necessidade. (Dunst \& Trivette, 1990; Ribeiro, 1999a; Silva, Ribeiro, Cardoso, Ramos, Carvalhosa, Dias, \& Gonçalves, 2003).

0 suporte social pode ser visto como informal, emocional, ou material (Singer \& Lord, 1984), suporte social psicológico e não-psicológico (fornecimento de informação versus suporte social tangível) (Cohen \& MacKay, 1984), o suporte social formal (grupos de amigos e familiares) e informal (organizações e instituições) (Dunst \& Trivette, 1990), o suporte social percebido e recebido (0 disponível versus o recebido relativamente ao pedido) e suporte social descrito e avaliado (presença de um tipo particular de assistência versus avaliação satisfatória ao pedido) (Cramer, Henderson, \& Scott, 1997).

Segundo Wilkins (2004) existem quatro tipos de disponibilidade de suporte social: 0 afeto; 0 suporte emocional e informador (afeto positivo, empatia, conselhos, orientação); a interação social positiva; e o suporte tangível (ajuda material ou assistência).

Para Dunst e Trivette (1990) existem cinco componentes interligadas com o suporte social: a componente constitucional (necessidades e a congruência entre a ajuda pedida e 0 suporte existente), a relacional (o estatuto familiar e profissional, o tamanho da rede social e a participação em organizações sociais), a funcional (suporte disponível e a qualidade do mesmo), a estrutural (envolve a proximidade física, a frequência de contactos, a proximidade psicológica, o nível da relação, reciprocidade e consistência), e da satisfação (envolve a relação entre a utilidade e a ajuda fornecida). Propõem ainda onze dimensões: 0 tamanho da rede social; a existência de relações sociais; a frequência de contactos sociais; a necessidade de suporte expressa pelo indivíduo; o tipo e a quantidade de suporte disponibilizado; a congruência entre o pedido e o suporte; a utilização ou recorrência à assistência; a dependência; a reciprocidade entre o suporte social recebido e o fornecido; a proximidade; e a satisfação entre o pedido de ajuda e o suporte recebido.

Para Matsukura, Marturano, e Oishi (2002), o suporte social envolve três componentes: os recursos de rede de suporte, o comportamento de suporte e as avaliações subjetivas do suporte. Identificam ainda a presença de três aspetos importantes, os relacionamentos sociais (quanto à 
existência, tipo e quantidade), o suporte social (quanto ao tipo, à fonte, à quantidade e qualidade), e a rede social (quanto ao tamanho, densidade, reciprocidade e intensidade).

A maioria dos autores definem a satisfação com o suporte social, como a avaliação cognitiva que 0 indivíduo faz em relação ao tipo e/ou qualidade do suporte social disponível (Trunzo \& Bernardine, 2003), podendo afirmar que a disponibilidade dos outros, medeia a relação entre 0 stress e a saúde (Button, 2008), e que a perceção da disponibilidade do suporte social se associa a uma adaptação fisiológica e psicológica do indivíduo (Wimberly, Carver, \& Antoni, 2008).

Diversas investigações comprovam os efeitos mediadores na proteção da saúde, na longevidade e na mortalidade, bem como ajuda a prevenir e recuperar a doença, e a aliviar o distress em situações de crise (Badoux, 2000; Bancila, 2006; Broadhead, Kaplan, James, Wagner, Schoenbach, Grimson, Heyden, Tibblin, \& Gehlbach, 1983; Coelho \& Ribeiro, 2000; Dunbar, Ford, \& Hunt, 1998; Ell, Nishimoto, Mediansky, Mantell, \& Hamovitch, 1992; Hanson, Isacsson, Janzon, \& Lindell, 1989; Hohaus \& Berah, 1996; Kessler, Price, \& Wortman, 1985; Magaya, Asner-Self, \& Schreiber, 2005; Ornelas, 1996; Quick, Saleh, Sime, \& Martin, 2006; Ribeiro, 2007; Ridder \& Schreurs, 1996; Rutter \& Quine, 1996; Schwarzer \& Leppin, 1991; Serra, 1999; Singer \& Lord, 1984; Southwick, Vythilingam, \& Charney, 2005; Wethingston \& Kessler, 1986; Wimberly, Carver, \& Antoni, 2008).

A satisfação profissional surge como parte da satisfação geral com a vida. Ganster, Fusilier, e Mayes (1986) verificaram que o suporte social tem um efeito moderador nas queixas somáticas, relativamente às condições de trabalho. Pinheiro e Ferreira, 2002 afirmam que tende a aumentar a autoestima, promover o humor positivo, o otimismo e a diminuir o stress, sentimentos de solidão e de fracasso.

A satisfação profissional resulta da experiência do indivíduo com o seu trabalho e desempenha um papel importante na progressão das suas metas, objetivos e projetos, pessoais e profissionais (Martins, 2005; Skaalvik \& Skaalvik, 2007), assim como se encontra intimamente relacionada com as gratificações auferidas e concretização de objetivos e aspirações, bem como com a saúde em geral, bem-estar e qualidade de vida (Serra, 1999).

A satisfação em contexto desportivo define-se como um estado afetivo positivo, que resulta da avaliação que 0 atleta faz das estruturas, dos processos e dos resultados, associados à prática e à experiência desportiva. Resulta assim da perceção que 0 atleta tem entre os ganhos e as perdas, entre 0 que deu e 0 que recebeu. É uma condição afetiva e está associada à forma como 0 atleta encara a sua carreira desportiva (Chelladurai \& Riemer, 1997; Riemer \& Chelladurai, 1998).

Segundo Riemer e Chelladurai (1998) a satisfação do atleta relaciona-se com resultados positivos e com os resultados desses processos; relaciona-se com os resultados desejados, a nível individual pela performance e pelo trabalho dos companheiros de equipa; e envolve tarefas, processos e resultados sociais.

No âmbito desportivo a satisfação do atleta engloba a relação da variável antecedente (fator pessoal) com a variável precedente (resultado) (Carron, Brawley, \& Widmeyer, 1985).

Podemos afirmar que a satisfação do atleta é uma variável de sucesso para o rendimento e para o desempenho desportivo (Chelladurai, 1980, 1990).

\section{MÉTODOS}

\section{Instrumentos:}

Um questionário sociodemográfico para recolher informações pessoais e profissionais e a Escala de Satisfação com o Suporte Social (ESSS), construída por Wethingson e Kessler e validada para a população portuguesa por Ribeiro em 1999. É m instrumento de autoresposta, que avalia quatro dimensões, a satisfação com amigos/amizades, a intimidade, a satisfação com a família e ati- 


\section{IMPACTO DA SATISFAÇÃO COM O APOIO SOCIAL EM ATLETAS}

vidades sociais, bem como um indicador global. A pontuação varia entre 15 e 75 pontos, onde notas mais altas correspondem a uma maior perceção de satisfação com o suporte social.

\section{Participantes:}

A amostra ficou concluída com 302 atletas seniores, profissionais de futebol, do género masculino, com idades compreendidas entre os 18 e os 38 anos de idade, maioritariamente de nacionalidade portuguesa, solteiros e sem filhos, e com o $3^{\circ}$ ciclo do ensino secundário. 41,7\% da III divisão competitiva, $21,5 \%$ da II divisão e $36,8 \%$ da 1a Liga. 9,9\% guarda-redes, $14,9 \%$ defesas centrais, $16,9 \%$ defesas laterais, $17,9 \%$ médios defensivos, $15,2 \%$ médios ofensivos, $11,3 \%$ médios ala, $7 \%$ pontas de lança e $7 \%$ avançados. 0 período de treino é maioritariamente no período noturno e fazem em média 5 treinos semanais. A maioria refere não sofrer de doenças ou lesões atuais e não dormir a sesta. Demoram em média 36 minutos nas deslocações de ida e volta para 0 treino, oscilando entre os 4 e 180 minutos.

\section{Análise Estatística:}

As hipóteses foram testadas com uma probabilidade de 95\%, resultando um nível de significância de $5 \%$, ( ) $\leq 0,05$, o que nos permite afirmar com 95\% de probabilidade caso se verifique a validade da hipótese em estudo, a existência de significância ou relação entre as variáveis, confirmando-se a probabilidade se inferior a 0,05 e rejeitando-se se superior ao valor.

Os pressupostos destes testes, nomeadamente o pressuposto de normalidade de distribuição e 0 pressuposto de homogeneidade de variâncias foram analisados com os testes de KolmogorovSmirnov e teste de Levene.

\section{RESULTADOS}

\section{Consistência Interna:}

Obtivemos um Alfa de Cronbach de 0,851 revelando uma boa consistência interna, valor semeIhante ao obtido pelo autor do instrumento $(0,85)$.

Verificámos que a idade influencia na satisfação com o suporte social (quadro 1). Atletas com idades entre 26-30 anos encontram-se mais satisfeitos com a intimidade, comparativamente aos com idade superior $(17,63$ vs 16,14$)$ (quadro 2 ).

Quadro 1 - Significância das diferenças: Idade

\begin{tabular}{|c|c|c|c|c|c|c|c|c|c|}
\hline & \multicolumn{2}{|c|}{ Até 20} & \multicolumn{2}{|c|}{$21-25$} & \multicolumn{2}{|c|}{$26-30$} & \multicolumn{2}{|c|}{$>30$} & \multirow[b]{2}{*}{ Sig. } \\
\hline & $\mathrm{M}$ & Dp & $\mathrm{M}$ & $\mathrm{Dp}$ & $\mathrm{M}$ & $\mathrm{Dp}$ & $\mathrm{M}$ & Dp & \\
\hline \multicolumn{10}{|l|}{ Suporte Social } \\
\hline Amizades & 20,19 & 3,46 & 20,49 & 3,13 & 20,32 & 3,16 & 19,30 & 3,00 & 0,360 \\
\hline Intimidade & 16,53 & 3,18 & 17,36 & 2,59 & 17,63 & 2,72 & 16,15 & 3,19 & $\mathbf{0 , 0 2 6}$ * \\
\hline Familia & 11,84 & 2,82 & 11,83 & 2,32 & 12,08 & 2,48 & 11,63 & 2,22 & 0,830 \\
\hline Atividades sociais & 10,27 & 2,37 & 10,83 & 2,19 & 10,38 & 2,54 & 10,30 & 2,55 & 0,307 \\
\hline Total & 58,83 & 9,03 & 60,51 & 8,15 & 60,41 & 8,57 & 57,37 & 8,74 & 0,225 \\
\hline
\end{tabular}


PSICOLOGÍA Y CAMBIO CIENTÍFICO EN POSITIVO

Quadro 2 - Teste de Tukey: idade vs satisfação com a intimidade

\begin{tabular}{lccc}
\hline Idade & $\mathrm{N}$ & \multicolumn{2}{c}{ Subset for alpha $=0.05$} \\
\cline { 3 - 4 } & & 1 & 2 \\
\hline 30 anos & 27 & $\mathbf{1 6 , 1 4}$ & 16,53 \\
Até 20 anos & 64 & 16,53 & 17,35 \\
$21-25$ anos & 138 & 17,35 & $\mathbf{1 7 , 6 3}$ \\
$26-30$ anos & 73 & & 0,176 \\
Sig. & & 0,115 & \\
\hline
\end{tabular}

0 escalão competitivo influencia na satisfação com o suporte social (quadro 3). A diferença é estatisticamente significativa entre seniores da III divisão e de 1a liga na satisfação com amizades, $(20,73$ vs 19,64) (quadro 4) e na satisfação com a intimidade (17,60 vs 16,61) (quadro 5). Na satisfação com a família, entre os seniores da III e II divisão $(12,41$ vs 11,35) (quadro 6) e na avaliação global entre os da III divisão e os de $1^{\text {a }}$ liga $(57,96$ vs 61,24$)$ (quadro 7$)$.

Quadro 3 - Significância das diferenças: escalão competitivo

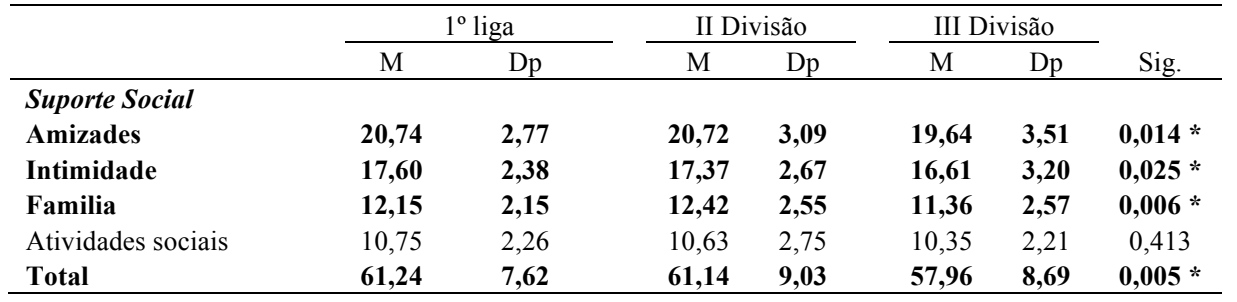

Quadro 4 - Teste de Tukey: escalão competitivo vs satisfação com as amizades

\begin{tabular}{lccc} 
Escalão Competitivo & $\mathrm{N}$ & \multicolumn{2}{c}{ Subset for alpha $=0.05$} \\
\cline { 3 - 4 } & & $\mathbf{1 9 , 6 4}$ & 2 \\
\hline Seniores III divisão & 126 & 20,72 & 20,72 \\
Seniores II divisão & 65 & & $\mathbf{2 0 , 7 3}$ \\
Seniores 1 ${ }^{\text {a Liga }}$ & 111 & 0,054 & 0,999 \\
Sig. & & & \\
\hline
\end{tabular}

Quadro 5 - Teste de Tukey: escalão competitivo vs satisfação com a intimidade

\begin{tabular}{lccc}
\hline Escalão Competitivo & $\mathrm{N}$ & \multicolumn{2}{c}{ Subset for alpha $=0.05$} \\
\cline { 3 - 4 } & & $\mathbf{1 6 , 6 1}$ & 2 \\
\hline Seniores III divisão & 126 & 17,36 & 17,36 \\
Seniores II divisão & 65 & & $\mathbf{1 7 , 6 0}$ \\
Seniores 1 ${ }^{\text {a }}$ Liga & 111 & 0,159 & 0,837 \\
Sig. & & \\
\hline
\end{tabular}




\section{IMPACTO DA SATISFAÇÃO COM O APOIO SOCIAL EM ATLETAS}

Quadro 6 - Teste de Tukey: escalão competitivo vs satisfação com a familia

\begin{tabular}{lccc}
\hline Escalão Competitivo & $\mathrm{N}$ & \multicolumn{2}{c}{ Subset for alpha $=0.05$} \\
\cline { 3 - 4 } & & $\mathbf{1 1 , 3 5}$ & 2 \\
\hline Seniores III divisão & 126 & 12,15 & 12,15 \\
Seniores 1 ${ }^{\text {a Liga }}$ & 111 & & $\mathbf{1 2 , 4 1}$ \\
Seniores II divisão & 65 & 0,066 & 0,741 \\
Sig. & & \\
\hline
\end{tabular}

Quadro 7 - Teste de Tukey: escalão competitivo vs avaliação global da satisfação com o suporte social

\begin{tabular}{lccc}
\hline Escalão Competitivo & $\mathrm{N}$ & \multicolumn{2}{c}{ Subset for alpha $=0.05$} \\
\cline { 3 - 4 } & & $\mathbf{5 7 , 9 6}$ & 2 \\
\hline Seniores III divisão & 126 & & 61,13 \\
Seniores II divisão & 65 & & $\mathbf{6 1 , 2 4}$ \\
Seniores 1ª Liga & 111 & 1,000 & 0,996 \\
Sig. & & & \\
\hline
\end{tabular}

Existe correlação positiva e moderada entre o período de treino e o escalão competitivo, $r=0,859$ e $p=0,000$ (1) (quadro 8). Atletas da 1ạ Liga treinam mais no período da manhã, os de II divisão no período da tarde, e de III divisão no período noturno (quadro 9).

Quadro 8 - Correlação Spearman's: período de treino e escalão competitivo

\begin{tabular}{rccc}
\hline & \multicolumn{3}{c}{ Periodo de treino habitual Escalão Competitivo } \\
\hline Spearman's rho Periodo de treino habitual $r$ & 1,000 & $\mathbf{0 , 8 5 9}^{* *}$ \\
& $p$ & $\cdot$ & $\mathbf{0 , 0 0 0}$ \\
$\mathrm{N}$ & 302 & 302 \\
\hline Escalão Competitivo & $r$ & $\mathbf{0 , 8 5 9}$ & 1,000 \\
& $p$ & $\mathbf{0 , 0 0 0}$ & $\cdot$ \\
$\mathrm{N}$ & 302 & 302 \\
\hline
\end{tabular}

Quadro 9 - Período de treino vs Escalão competitivo

\begin{tabular}{llcccc}
\hline & & \multicolumn{3}{c}{ Periodo de treino habitual } & \\
\cline { 3 - 5 } & & Manhã & Tarde & Noite & Total \\
\hline Escalão Competitivo & Seniores 1 $1^{\text {a }}$ Liga & $\mathbf{7 6}$ & 34 & 1 & 111 \\
& Seniores II divisão & 0 & $\mathbf{4 9}$ & 16 & 65 \\
& Seniores III divisão & 3 & 6 & $\mathbf{1 1 7}$ & 126 \\
Total & & 79 & 89 & 134 & 302 \\
\hline
\end{tabular}

0 período de treino influencia a satisfação com o suporte social (quadro 10). A diferença é estatisticamente significativa entre os atletas que treinam à noite e os que treinam à tarde, sentindo estes maior satisfação com amizades (20,78 vs 19,77$)$ (quadro 11), com a família, (12,50 vs 11,35$)$ (quadro 12) e na avaliação global $(61,46$ vs 58,14$)$ (quadro 13). 
Quadro 10 - Significância das diferenças: período de treino

\begin{tabular}{|c|c|c|c|c|c|c|c|}
\hline & \multicolumn{2}{|c|}{ Manhã } & \multicolumn{2}{|c|}{ Tarde } & \multicolumn{2}{|c|}{ Noite } & \multirow[b]{2}{*}{ Sig. } \\
\hline & $\mathrm{M}$ & $\mathrm{Dp}$ & $\mathrm{M}$ & $\mathrm{Dp}$ & $\mathrm{M}$ & $\mathrm{Dp}$ & \\
\hline \multicolumn{8}{|l|}{ Suporte Social } \\
\hline Amizades & 20,56 & 2,77 & 20,79 & 3,17 & 19,78 & 3,40 & $0,046 *$ \\
\hline Intimidade & 17,48 & 2,44 & 17,49 & 2,71 & 16,70 & 3,09 & 0,057 \\
\hline Familia & 12,06 & 2,25 & 12,51 & 2,32 & 11,35 & 2,56 & $0,002 *$ \\
\hline Atividades sociais & 10,84 & 2,33 & 10,67 & 2,42 & 10,31 & 2,32 & 0,252 \\
\hline Total & 60,94 & 7,98 & 61,46 & 8,72 & 58,14 & 8,43 & $\mathbf{0 , 0 0 7}$ * \\
\hline
\end{tabular}

Quadro 11 - Teste de Tukey: período de treino vs satisfação com as amizades

\begin{tabular}{|c|c|c|c|}
\hline \multirow[t]{2}{*}{ Periodo de treinos habituais } & \multirow[t]{2}{*}{$\mathrm{N}$} & \multicolumn{2}{|c|}{ Subset for alpha $=0.05$} \\
\hline & & 1 & 2 \\
\hline Noite & 134 & 19,77 & \\
\hline Manhã & 79 & 20,55 & 20,55 \\
\hline Tarde & 89 & & 20,78 \\
\hline Sig. & & 0,073 & 0,731 \\
\hline
\end{tabular}

Quadro12 - Teste de Tukey: período de treino vs satisfação com a familia

\begin{tabular}{lccc}
\hline Periodo de treinos habituais & $\mathrm{N}$ & \multicolumn{2}{c}{ Subset for alpha $=0.05$} \\
\cline { 3 - 4 } & & 1 & 2 \\
\hline Noite & 134 & $\mathbf{1 1 , 3 5}$ & 12,06 \\
Manhã & 79 & 12,06 & $\mathbf{1 2 , 5 0}$ \\
Tarde & 89 & & 0,104 \\
Sig. & & & 0,414 \\
\hline
\end{tabular}

Quadro13 - Teste de Tukey: período de treino vs avaliação global da satisfação com o suporte social

\begin{tabular}{lccc}
\hline Periodo de treinos habituais & $\mathrm{N}$ & \multicolumn{2}{c}{ Subset for alpha $=0.05$} \\
\cline { 3 - 4 } & & 1 & 2 \\
\hline Noite & 134 & $\mathbf{5 8 , 1 4}$ & 60,93 \\
Manhã & 79 & 60,93 & $\mathbf{6 1 , 4 6}$ \\
Tarde & 89 & & 0,903 \\
Sig. & & 0,057 & \\
\hline
\end{tabular}

0 tempo gasto em deslocações para 0 treino influencia a satisfação com 0 suporte social na satisfação com a família (quadro 14) (11,40 vs 12,34) (quadro 15).

Quadro 14 - Significância das diferenças: tempo gasto na deslocação para o treino

\begin{tabular}{|c|c|c|c|c|c|c|c|}
\hline & \multicolumn{2}{|c|}{$15 \mathrm{~m}$} & \multicolumn{2}{|c|}{1 hora } & \multicolumn{2}{|c|}{$>1$ hora } & \multirow[b]{2}{*}{ Sig. } \\
\hline & M & $\mathrm{Dp}$ & M & $\mathrm{Dp}$ & $\mathrm{M}$ & $\mathrm{Dp}$ & \\
\hline \multicolumn{8}{|l|}{ Suporte Social } \\
\hline Amizades & 19,92 & 3,22 & 20,40 & 3,20 & 20,71 & 3,13 & 0,331 \\
\hline Intimidade & 16,94 & 2,84 & 17,22 & 2,87 & 17,32 & 2,72 & 0,676 \\
\hline Familia & 11,41 & 2,49 & 12,07 & 2,46 & 12,34 & 2,18 & 0,047 * \\
\hline Atividades sociais & 10,36 & 2,27 & 10,65 & 2,38 & 10,71 & 2,48 & 0,573 \\
\hline Total & 58,63 & 8,54 & 60,34 & 8,64 & 61,08 & 7,72 & 0,180 \\
\hline
\end{tabular}




\section{IMPACTO DA SATISFAÇÃO COM O APOIO SOCIAL EM ATLETAS}

Quadro 15 - Teste de Tukey: tempo de deslocação para o treino vs satisfação com a família

\begin{tabular}{lccc}
\hline Deslocação & $\mathrm{N}$ & \multicolumn{2}{c}{ Subset for alpha $=0.05$} \\
\cline { 3 - 4 } & & $\mathbf{1 1 , 4 0}$ & 2 \\
\hline Até 15minutos & 103 & 12,06 & 12,06 \\
15 minutos a 1 hora & 161 & & $\mathbf{1 2 , 3 4}$ \\
$>$ 1hora & 38 & 0,059 & 0,059 \\
Sig. & & & \\
\hline
\end{tabular}

A posição de jogo influencia na satisfação com o suporte social (quadro 16). Na satisfação com as amizades as diferenças encontram-se entre ponta de lança, e os guarda-redes e avançados, $(21,28$ e 21,23 vs 18,42) (quadro 17). Na satisfação com as atividades sociais as diferenças encontram-se entre ponta de lança, e os guarda redes, avançados e médios (11,24 11,04 11,00 vs 9,09) (quadro 18).

Quadro 16 - Significância das diferenças: posição de jogo

\begin{tabular}{lc}
\hline & Sig. \\
\hline Satisfação social & $\mathbf{0 , 0 3 5 *}$ \\
Amizades & 0,142 \\
Intimidade & 0,525 \\
Familia & $\mathbf{0 , 0 2 7} *$ \\
Atividades Sociais & 0,165 \\
Total & \\
\hline
\end{tabular}

Quadro 17- Teste de Tukey: posição de jogo vs satisfação com as amizades

\begin{tabular}{lccc}
\hline & $\mathrm{N}$ & \multicolumn{2}{c}{ Subset for alpha $=0.05$} \\
\cline { 3 - 4 } & & $\mathbf{1 8 , 4 2}$ & 2 \\
\hline Ponta de lança & 21 & 19,66 & 19,66 \\
Defesa lateral & 51 & 20,11 & 20,11 \\
Médio seniors & 54 & 20,26 & 20,26 \\
Defesa central & 45 & 20,58 & 20,58 \\
Médio ofensivo & 46 & 20,73 & 20,73 \\
Médio ala & 34 & & $\mathbf{2 1 , 2 3}$ \\
Guarda redes & 30 & & $\mathbf{2 1 , 2 8}$ \\
Avançado & 21 & 0,061 & 0,422 \\
Sig. & & & \\
\hline
\end{tabular}

Quadro 18 - Teste de Tukey: posição de jogo vs atividades sociais

\begin{tabular}{lccc}
\hline & $\mathrm{N}$ & \multicolumn{2}{c}{ Subset for alpha $=0.05$} \\
\cline { 3 - 4 } & & $\mathbf{9 , 0 9}$ & 2 \\
\hline Ponta de lança & 21 & 10,29 & 10,29 \\
Médio ala & 34 & 10,37 & 10,37 \\
Defesa central & 45 & 10,39 & 10,39 \\
Médio ofensivo & 46 & 10,45 & 10,45 \\
Defesa lateral & 51 & & $\mathbf{1 1 , 0 0}$ \\
Guarda redes & 30 & & $\mathbf{1 1 , 0 4}$ \\
Avançado & 21 & & $\mathbf{1 1 , 2 4}$ \\
Médio defensive & 54 & & 0,709 \\
Sig. & & 0,251 & \\
\hline
\end{tabular}




\section{DISCUSSOES E CONCLUSÕES}

Os atletas profissionais de futebol possuem níveis adequados de percepção da satisfação com o suporte social, revelando os resultados que é influenciada pela idade, lesões/doenças, escalão competitivo, período de treino, número de treinos semanais, posição de jogo e tempo gasto nas deslocações para 0 treino.

Atletas com idades entre os 26 e os 30 anos de idade apresentam uma maior satisfação com a intimidade, comparativamente aos atletas com idade superior a 30 anos. A intimidade é uma dimensão muito pessoal que necessita de ser satisfeita, refletindo a especificidade das relações interpessoais e intrapessoais. Na maioria são os jovens adultos, solteiros, que dão importância à vivência de relações íntimas, concebem relações românticas, envolvidas na paixão, atração física, sexualidade, amor, desejo e excitação. Uma das principais tarefas do jovem adulto é a procura de intimidade (Arnett, 2000; Perlman \& Duck, 1987). A intimidade relaciona-se ainda positivamente com a satisfação com a vida. A manutenção de relações íntimas é uma necessidade humana, presente em todas as culturas, sendo a qualidade das relações, determinantes para o bem-estar físico, emocional e social (Laurenceau, Riviera, Shaffer, \& Pietromonaco, 2004). Os resultados corroboram estudos onde atletas mais velhos tendem a preocupar-se mais em trabalhar na modalidade, estando mais interessados na demonstração da aprendizagem e com o seu domínio ou tarefa, num ambiente de profissionalização (Terry, Galambos, West \& lizuka, 2007). Folkman e Lazarus (1988) referem ainda que padrões de confronto estão adaptados à idade, onde os mais novos recorrem a estratégias mais ativas e interpessoais comparativamente aos mais velhos.

Dado o escasso número de atletas que revelou sofrer de lesões ou doenças, o resultado não foi significativo, no entanto além terem implicações na diminuição do rendimento desportivo, podem ainda interferir na perceção da satisfação com suporte social (Marques \& Oliveira, 2001; Pastre, Carvalho, Monteiro, Júnior, \& Padovani, 2005). 0 escalão competitivo influencia a satisfação com 0 suporte social, sendo os atletas de 1ํㅡㄴ Liga os que obtêm maior satisfação com amizades e intimidade. Por sua vez, são os atletas da II divisão os que obtêm maior satisfação com a família. Corroborando outros estudos, que concluíram que os atletas de nível competitivo superior apresentam uma maior utilização de estratégias de coping comparativamente aos de nível competitivo inferior (Golby \& Sheard, 2004), determinantes para alcançar elevados níveis de rendimento, em qualquer modalidade desportiva (Dale \& Weinberg, 1989; Maslach \& Jackson, 1981; Raedeke, 1997; Smith, 1986; Vealey, Armstrong, Comar, \& Greenleaf, 1998; Vealey, Udry, Zirmmermman, \& Soliday, 1992). Outras investigações demonstraram que atletas de escalão competitivo superior possuem valores mais elevados de autoconfiança, comparativamente aos de nível competitivo inferior (Bush, Salmela, \& Demers, 2001; Cruz, 1996; Davis \& Cox, 2002; Gould, Eklund, \& Jackson, 1992; Rodrigues \& Cruz, 1997). Existe uma relação estatisticamente significativa entre o período de treino e 0 escalão competitivo, havendo uma proporção significativamente mais elevada de atletas da 1 a liga que treinam no período da manhã, de atletas da II divisão que treinam no período da tarde e de atletas da III divisão que treinam no período noturno. Estes resultados vão ao encontro da prática de treino habitual em Portugal, para estes escalões competitivos.

0 número de treinos semanais não influenciou a satisfação com o suporte social podendo afirmar, que a média de 5 treinos semanais que os atletas fazem, se ajustam às necessidades físicas, psíquicas e sociais dos atletas. 0 período de treino influência a satisfação com o suporte social, onde atletas que treinam no período noturno revelam valores inferiores de satisfação com amizades e com a família, comparativamente ao que treinam no período da tarde, sentindo estes uma melhor perceção destes suportes, corroborando estudos de Zuzanek e Mannel (2000). Estudos revelam que uma prática regular e bem orientada das atividades desportivas, contribuem para uma melhor per- 


\section{IMPACTO DA SATISFAÇÃO COM O APOIO SOCIAL EM ATLETAS}

ceção da imagem corporal e uma auto-estima mais elevada, além de melhorar o bem-estar psicológico, através da redução dos níveis de stress, de ansiedade e de depressão (Mello, Boscolo, Esteves, \& Tufit, 2005; Samulski, Costa, Amaparo, \& Silva, 2009).

A posição de jogo influencia a satisfação com o suporte social, onde guarda-redes e avançados sentem uma maior satisfação com as amizades, comparativamente aos atletas ponta de lança. Guarda-redes, avançados e médios ofensivos, obtêm maior satisfação nas atividades sociais, comparativamente aos atletas ponta de lança. 0 tempo de deslocação de ida de volta para 0 treino influencia satisfação com o suporte social, ao nível da satisfação com família. Verificamos que são os atletas da III divisão os que demoram mais tempo em deslocações para os treinos, seguidos dos atletas de II divisão e 1a liga.

Conclui-se que os atletas profissionais possuem níveis adequados de percepção da satisfação com o suporte social, e esta é influenciada pela idade, lesões ou doenças, escalão competitivo, período de treino, número de treinos semanais, posição de jogo e tempo gasto nas deslocações para o treino, permitindo delinear programas preventivos e de intervenção durante as fases de pré-competição e competição.

Uma perceção adequada do suporte social desempenha um papel importante na progressão de metas, objetivos, projetos pessoais e profissionais. Neste sentido, a perceção que 0 atleta tem do suporte social é passível de afetar e influenciar o seu equilíbrio global, sendo vista como uma variável de sucesso para o rendimento e desempenho desportivo.

\section{REFERÊNCIAS}

Arnett, J. (2000). Emerging adulthood: A theory of development from the late teens through the twenties. American Psychologist, 55, 5, 469-480.

Badoux, A. (2000). Social support in healthy and psychologically distressed French populations. Psychology, Health \& Medicine, 5, 2, 142 - 153.

Bancila, D. (2006). The association of interpersonal stress with psychological distress in Romania. European Psychologist, 11, 1, 39-49.

Berkman, L. (1984). Assessing the physical health effects of social networks and social support. Annual Review of Public Health, 5, 413-432.

Broadhead, W., Kaplan, B., James, S., Wagner, E., Schoenbach, V., Grimson, R., Heyden, S., Tibblin, G., \& Gehlbach, S. (1983). The epidemiologic evidence for a relationship between social support and health. American Journal of Epidemiology, 117, 5, 521-537.

Bruhn, J. \& Philips, B. (1984). Measuring social suport: a synthesis of current approaches. Journal of Behavioral Medicine, 7, 2, 151-169.

Bush, N., Salmela, J. \& Demers, I. (2001). The Ottawa Mental Skills Assessment Tool (OMSAT-3). The Sport Psychologist, 15, 1-19.

Button, L. (2008). Effect of social support and coping strategies on the relationship between health care-related occupational stress and health. Journal of Research in Nursing, 13, 6, 498-524.

Carron, A., Brawley, L., \& Widmeyer, W. (1985). The development of an instrument to measure cohesion in sport teams: The Group Environment Questionnaire. Journal of Sport \& Exercise Psychology, 7, 244-66.

Cassel, J. (1976). The contribution of the social environment to host resistance. American Journal of Epidemiology, 104, 2, 107-123.

Chelladurai, P. (1980). Leadership in sport organizations. Canadian Journal of Applied Sport Sciences, 5, 4, 226-231.

Chelladurai, P. (1990). Leadership in sports: A review. International Journal of Sport Psychology, 21, 
328-354.

Chelladurai, P., \& Riemer, H. (1997). A classification of the facets of athlete satisfaction. Journal Sport Management, 11, 2, 133-59.

Cobb, S. (1976). Social support as a moderator of life stress. Psychosomatic Medicine, 38, 5, 300314.

Coelho, M. \& Ribeiro, J. (2000). Influência do suporte social e do coping sobre a percepção subjectiva de bem-estar em mulheres submetidas a cirurgia cardíaca. Psicologia, Saúde \& Doenças, 1, 1, 79-87.

Cohen, S. (1988). Psychosocial models of the role of social support in the etiology of psysical disease. Health Psychology, 7, 3, 269-297.

Cohen, S., \& McKay, G. (1984). Social support, stress, and the buffering hypothesis: A theoretical analysis. In A. Baum, J. E. Singer, \& S. E. Taylor (Eds.), Handbook of psychology and health, IV. Hillsdale, NJ: Erlbaum.

Cramer, D., Henderson, S., \& Scott, R. (1997). Mental health and desired social support: a four-wave panel study. Journal of Social and Personal Relationships, 14, 6, 761-775.

Cruz, J. (1996). Stress, ansiedade e competências psicológicas nos atletas de elite e de alta competição: Um estudo da sua relação e impacto no rendimento e no sucesso desportivo. Psicologia: Teoria, Investigação e Prática, 1, 161-192.

Dale, J., \& Weinberg, R. (1989). The relationship between coaches leadership style and burnout. The Sport Psychologist, 3, 1-13.

Davis, J. \& Cox, R. (2002). Interpreting direction of anxiety within Hanin's individual zone of optimal functioning. Journal of Applied Sport Psychology, 14, 43-52.

Dunbar, M., Ford, G., \& Hunt, K. (1998). Why is the receipt social support associated with increased psychosocial distress? An examination of three hypotheses. Psychology and Health, 13, 527544.

Dunst, C., \& Trivette, C. (1990). Assessment of social support in early intervention programs. In S. Meisels, \& J. Shonkoff (Eds.), Handbook of early childhood intervention. New York: Cambridge University Press, 326-349.

Ell, K., Nishimoto, R., Mediansky, L., Mantell, J., \& Hamovitch, M. (1992). Social relations, social support and survival among patients with cancer. Journal of Psychosomatic Research, 36, 6 , $531-541$.

Ganster, D., Fusilier, M., \& Mayes, B. (1986). Role of social support in the experience of stress at work. Journal of Applied Psychology, 71, 1, 102-110.

Golby, J. \& Sheard, M. (2004). Mental toughness and hardiness at different levels of rugby league. Personality and Individual Differences, 37, 933-942.

Gould, D., Eklund, R., \& Jackson, S. (1992). 1988 U.S. Olympic wrestling excellence: I. Mental preparation, precompetitive cognition, and affect. The Sport Psychologist, 6, 358-382.

Hanson, B., Isacsson, S., Janzon, L., \& Lindell, S. (1989). Social network and social support influence mortality in elderly mem. American Journal of Epidemiology, 130, 1, 100-111.

Hohaus, L., \& Berah, E. (1996). Stress, achievement, marriage and social support: effects on the psychological well-being of physicians entering midlife/mid-career. Psychology and Health, 11, 715- 731.

Kaplan, B., Cassel, J., \& Gore, S. (1977). Social support and health. Medical Care, 15, 5, 47-58.

Kessler, R., Price, R., \& Wortman, C. (1985). Social factors in psychopathology: stress, social support, and coping process. Annual Review of Psychology, 36, 531-572.

Laurenceau, J., Rivera, L., Schaffer, A. \& Pietromonaco, P. (2004). Intimacy as an interpersonal pro- 
cess: Current status and future directions. In Mashek, D. \& Aron, A. (Eds.), Handbook of Closeness and Intimacy. New Jersey: Laurence Erlbaum Associates.

Magaya, L., Asner-Self, K. \& Schreiber, J. (2005). Stresse and coping strategies among Zimbabwean adolescents. British Journal of Educational Psychology, 75, 661-671.

Marques, A., \& Oliveira, J. (2001). 0 treino e a competição dos mais jovens: rendimento versus saúde. Revista Portuguesa de Ciências do Desporto, 1.

Martins, M. (2005). Sintomas de stress em professores das primeiras séries do ensino fundamental: Um estudo exploratório. Dissertação de Mestrado. Lisboa: Universidade Lusófona de Humanidades e Tecnologias.

Maslach, C., \& Jackson, S. (1981). The Measurement of Experienced Burnout. Journal of Occupational Behavior, 2, 2, 99-113.

Matsukura, T., Maturano, E., \& Oishi, J. (2002). 0 questionário de suporte social (SSQ): estudos de adaptação para o português. Revista Latino-Americana de Enfermagem, 10, 5, 675-681.

Mello, M., Boscolo, R., Esteves, A., \& Tufik, S. (2005). 0 exercício físico e os aspectos psicobiológicos. Revista Brasileira de Medicina do Esporte, 11, 3, 203-207.

Ornelas, J. (1994). Suporte social: origens, conceitos e áreas de intervenção. Análise Psicológica, 2 , 12, 333-339.

Ornelas, J. (1996). Suporte social e doença mental. Análise Psicológica, 14, 2, 3, 263-268.

Pastre, C., Carvalho, G., Monteiro, H., Júnior, J., \& Padovani, C. (2005). Lesões desportivas na elite do atletismo brasileiro: estudo a partir de morbidade referida. Revista Brasileira de Medicina do Esporte, 11, 1, 43-47.

Perlman, D., \& Duck, S. (Eds.). (1987). Intimate Relationships. Development, Dynamics, and Deterioration. Beverly Hills: Sage Publications.

Quick, J., Saleh, K., Sime, W. \& Martin, W. (2006). Stress management skills for strong leadership: Is it worth dying for? The Journal of Bone \& Joint Surgery, 88, A, 1, 217-225.

Raedeke, T. (1997). Is Athlete burnout more than just stress? A sport commitment perspective. Journal of Sport and Exercise Psychology, 19, 4, 396-417.

Ribeiro, J. (1999). Escala de Satisfação com o Suporte Social. Análise Psicológica, 3, 17, 547-558.

Ridder, D., \& Schreurs, K. (1996). Coping, social support and chronic disease: a research agenda. Psychology, Health \& Medicine, 1, 71-82.

Riemer, H., \& Chelladurai, P. (1998). Development of the Athlete Satisfaction Questionnaire (ASQ). Journal of Sport and Exercise Psychology, 20, 2, 127-156.

Rodrigues, R., \& Cruz, J. (1997). Auto- -confiança, ansiedade e rendimento na natação de alta competição: Estudo com os atletas de elite nacional. Psicologia: Teoria, investigação e prática, 3, 491-521.

Rutter, D., \& Quine, L. (1996). Social psychological mediators of the relationship between demographic factors and health outcomes: a theoretical model and some preliminary data. Psychology and Health, 11, 5-22.

Samulski, D., Costa, I., Amaparo, L., \& Silva, L. (2009). Atividade física, saúde e qualidade de vida. Psicologia do Esporte: conceitos e novas perspectivas, 357-382.

Serra, A. (1999). O stress na vida de todos os dias. Coimbra: Gráfica de Coimbra.

Silva, I., Ribeiro, J., Cardoso, H., Ramos, H., Carvalhosa, S., Dias, S., \& Gonçalves, A. (2003), Efeitos do apoio social na qualidade de vida, controlo metabólico e desenvolvimento de complicações crónicas em indivíduos com diabetes. Psicologia, Saúde e Doenças, 4, 1, 21-32.

Singer, J., \& Lord, D. (1984). The role of social support in coping with chronic or life-threatning illness. In A. Baum, S. Taylor, \& J. Singer (Eds.), Handbook of psychology and health, IV, 269-278. 
New Jersey: Laurence Erlbaum Associates, Inc., Publishers.

Skaalvik, E., \& Skaalvik, S.(2007). Dimensions of teacher self-efficacy and relations with strain factors, perceived collective teacher efficacy, and teacher burnout. Journal of Educational Psychology, 99, 3, 611-625.

Smith, R. (1986). Toward a cognitive-affective model of athletic burnout. Journal of Sport Psychology, 8, 36-50.

Southwick, S., Vythilingam, M., \& Charney, D. (2005). The psychobiology of depression and resilience to stress: Implications for prevention and treatment. Annual Review of Clinical Psychology, 1, 255-291.

Taylor, S. (1990). Health psychology: the science and the field. American Psychologist, 45, 40-50.

Terry, P., Galambos, S., West, J., \& lizuka, C. (2007). Psychological correlates of training load among athletes. Australian Psychological Society.

Trunzo, J., \& Bernardine M. (2003). Social Support as a Mediator of Optimism and Distress in Breast Cancer Survivors. Journal of Consulting \& Clinical Psychology, 71, 4, 805.

Vealey, R., Armstrong, L., Comar, W., \& Greenleaf, C. (1998). Influence of perceived coaching behaviours on burnout and competitive anxiety in female college athletes. Journal of Applied Sport Psychology, 10, 297-318.

Vealey, R., Udry E., Zimmerman, V., \& Soliday, J. (1992). Interpersonal and situational predictors of coaching burnout. Journal of Sports and Exercise Pshychology, 14, 40-58.

Wethingston, E., \& Kessler, R. (1986). Perceived support, received support, and adjustment to stressfull life evets. Journal of Health and Social behavior, 27, 78-89.

Wilkins, K. (2004). Bipolar I discorder, social support and work. Supplement to the Health Repports, 15, 23-32.

Wimberly, S., Carver, C. \& Antoni, M. (2008). Effects of optimism, interpersonal relationships, and distress on psychosexual well-being among women with early stage breast cancer. Psychology and Health, 23, 1, 57-72.

Zuzanek, J., \& Mannell, R. (2000). Effects of physically active leisure, social support, work stress, and chronic stress on mental health and physical health: A longitudinal perspective. Faculty of Applied Health Sciences. University of Waterloo. 
